\title{
Floquet Mechanism for Non-Abelian Fractional Quantum Hall States
}

\author{
Ching Hua Lee, ${ }^{1,2}$ Wen Wei Ho, ${ }^{3}$ Bo Yang, ${ }^{1,4}$ Jiangbin Gong, ${ }^{2}$ and Zlatko Papic ${ }^{5}$ \\ ${ }^{1}$ Institute of High Performance Computing, A*STAR, Singapore, 138632 \\ ${ }^{2}$ Department of Physics, National University of Singapore, Singapore, 117542 \\ ${ }^{3}$ Department of Physics, Harvard University, Cambridge, Massachusetts 02138, USA \\ ${ }^{4}$ Division of Physics and Applied Physics, Nanyang Technological University, Singapore, 637371 \\ ${ }^{5}$ School of Physics and Astronomy, University of Leeds, Leeds LS2 9JT, United Kingdom
}

(Received 24 May 2018; revised manuscript received 17 September 2018; published 4 December 2018)

\begin{abstract}
Three-body correlations, which arise between spin-polarized electrons in the first excited Landau level, are believed to play a key role in the emergence of enigmatic non-Abelian fractional quantum Hall (FQH) effects. Inspired by recent advances in Floquet engineering, we investigate periodic driving of anisotropic two-body interactions as a route for controllably creating and tuning effective three-body interactions in the FQH regime. We develop an analytic formalism to describe this Floquet-FQH protocol, which is distinct from previous approaches that instead focus on band structure engineering via modulation of single-particle hopping terms. By systematically analyzing the resulting interactions using generalized pseudopotentials, we show that our Floquet-FQH approach leads to repulsive as well as attractive threebody interactions that are highly tunable and support a variety of non-Abelian multicomponent FQH states. Finally, we propose an implementation of the protocol in optically dressed ultracold polar molecules with modulated Rabi frequencies.
\end{abstract}

DOI: 10.1103/PhysRevLett.121.237401

Topological phases exhibit enticing prospects for faulttolerant quantum computation [1,2] owing to their exotic quasiparticle excitations [3-5]. These phases are believed to arise from an interplay between the Coulomb interaction, Landau level quantization and complete spin polarization in 2D electronic systems [6], as suggested by the observation of even-denominator fractional quantum Hall $(\mathrm{FQH})$ plateaus in semiconductors [7] and recently in bilayer graphene $[8,9]$. The unexpected even-denominator plateaus are explained by adiabatic continuity [10-12] between the underlying gapped many-electron state and the ground state of a system with special three-body electronic interactions $[13,14]$. Such three-body interactions condense the electrons into a strongly correlated quantum state where they fractionalize into non-Abelian Ising anyons [3]. More generally, multibody interactions are anticipated to give rise to other types of non-Abelian anyons [15-17].

Conventionally, effective three-body interactions arise due to Coulomb interactions and virtual excitations between Landau levels (LLs) [18-22], a process suppressed by the LL splitting in a magnetic field, given by the cyclotron energy $\hbar \omega_{c}=[(\hbar e B) /(m c)]$. At the same time, the incompressibility gap, which determines the stability of a FQH state, scales as $e^{2} / \epsilon \ell_{B}$, where $\ell_{B}=\sqrt{\hbar / e B}$ is the magnetic length. Thus, the effect of three-body interactions can typically only be enhanced at the expense of reducing the energy gap, which weakens the FQH state.

Inspired by recent progress in "Floquet engineering" [23-28], we propose an alternative method to realize effective three-body interactions and hence stabilize various non-Abelian $\mathrm{FQH}$ states. Our approach consists of periodically modulating (two-body) interactions, specifically the repulsion between spatially separated electrons. The key to our idea is the noncommutativity of the Girvin-MacDonald-Platzman (GMP) algebra [29,30] describing the electron density operators projected to a $\mathrm{LL}$, which is the defining property of both continuum $\mathrm{FQH}$ states [29] and their lattice analogs, the fractional Chern insulators (FCIs) [31]. We show that, owing to this algebra, the effective, static Hamiltonian that arises when a generic anisotropic $\mathrm{FQH}$ system is driven at high frequencies contains a rich set of many-body interactions which scale with the inverse driving frequency, rather than the LL gap. In particular, desired three-body multicomponent (spin) interactions can be engineered by time modulation of realistic two-body interactions. More generally, we systematically analyze the interactions resulting from our "Floquet-FQH" protocol using the framework of generalized pseudopotentials [32], and show that the drive can also generate attractive three-body interactions.

Finally, we discuss a realistic implementation of the Floquet-FQH protocol in ultracold molecules optically dressed with modulated Rabi frequencies, whose static version was previously established to host FCI states [33]. We note that our approach is conceptually different from previous Floquet proposals [34-37] and experiments [38] which focused on topological band engineering via modulation of (single-body) kinetic terms; it is also distinct from works [39-42], which modulated on-site two-body 
interactions to probe tunneling phenomena, Mott and superfluid phases.

Two key inspirations.-(i) A defining feature of $\mathrm{FQH}$ systems [29] is the GMP algebra

$$
\left[\bar{\rho}_{\mathbf{q}}^{\sigma}, \bar{\rho}_{\mathbf{q}^{\prime}}^{\sigma^{\prime}}\right]=2 i \delta_{\sigma, \sigma^{\prime}} \sin \frac{\hat{\mathbf{z}} \cdot\left(\mathbf{q} \times \mathbf{q}^{\prime}\right) \ell_{B}^{2}}{2} \bar{\rho}_{\mathbf{q}+\mathbf{q}^{\prime}}^{\sigma},
$$

obeyed by the density operators $\bar{\rho}_{\mathbf{q}}^{\sigma} \equiv \mathcal{P} \rho_{\mathbf{q}}^{\sigma} \mathcal{P}=\sum_{j} e^{i \mathbf{q} \cdot \mathbf{R}_{j}^{\sigma}}$, projected to a given LL via $\mathcal{P}$. Here, $R_{j}^{\sigma, a} \equiv r_{j}^{\sigma, a}+$ $\ell_{B}^{2} \epsilon^{a b} \Pi_{j, b}^{\sigma}$ denote the guiding-center coordinates of the $j$ th particle with spin $\sigma$ [6], $\epsilon^{a b}$ is the antisymmetric tensor $(a=x, y)$. Note that $R_{j}^{\sigma, a}$ differ from the position coordinates $r_{j}^{\sigma}$ by the canonical momentum $\Pi_{j}^{\sigma, a}=q_{j}^{\sigma, a}-e A^{a}$ in a magnetic field $B=\epsilon^{a b} \partial_{a} A_{b}$, and thus $R_{j}^{\sigma, a}$ do not commute. The same density algebra, Eq. (1), is also obeyed by the FCIs in the thermodynamic and long-wavelength limit $[31,43,44]$, with magnetic field replaced by mean Berry curvature [44]. We will work in this limit, and will henceforth not distinguish between FQH and FCI. Our Floquet-FQH approach is based on the observation that the repeated application of the GMP algebra produces $(2 N-1)$-body terms from the commutator of two $N$-body terms. In particular, the commutator of two two-body terms yields a potentially desirable three-body term.

(ii) At high frequencies $\Omega=2 \pi / T$, the stroboscopic dynamics of a periodically driven system, $H(t)=$ $\sum_{l} e^{i l \Omega t} H_{l}$, can be captured by the static effective Hamiltonian

$$
H^{\mathrm{eff}}=H_{0}+\frac{1}{\hbar \Omega} \sum_{l} \frac{1}{l}\left[H_{l}, H_{-l}\right]+\cdots,
$$

obtained, e.g., from the Magnus or other equivalent highfrequency expansions [24,45,46]. Most saliently, Eq. (2) involves commutators which represent the renormalizing effects of the drive on the interactions. Thus, we see that dynamically modulating a FQH system, combined with the structure of the GMP algebra in (i), is a natural way to realize higher-body interaction terms, Fig. 1(a).

Importance of anisotropy. - A necessary condition for our Floquet-FQH approach is that the commutators in Eq. (2) do not vanish (this does happen if the system is rotationally symmetric). Such commutators, however, can be shown to generically survive in anisotropic FQH systems. Our protocol is thus targeted at $\mathrm{FQH}$ systems with anisotropic interactions; note that this is not a major restriction because anisotropy is ubiquitous in many setups: it can be induced by tilting the magnetic field [47-49], and it is intrinsically large in FCIs [32].

We remark that the anisotropy of FQH-FCI systems can be quantified using standard Haldane pseudopotentials (PPs) and their generalizations to $N$-particle interactions with internal degrees of freedom [50-52], which we briefly
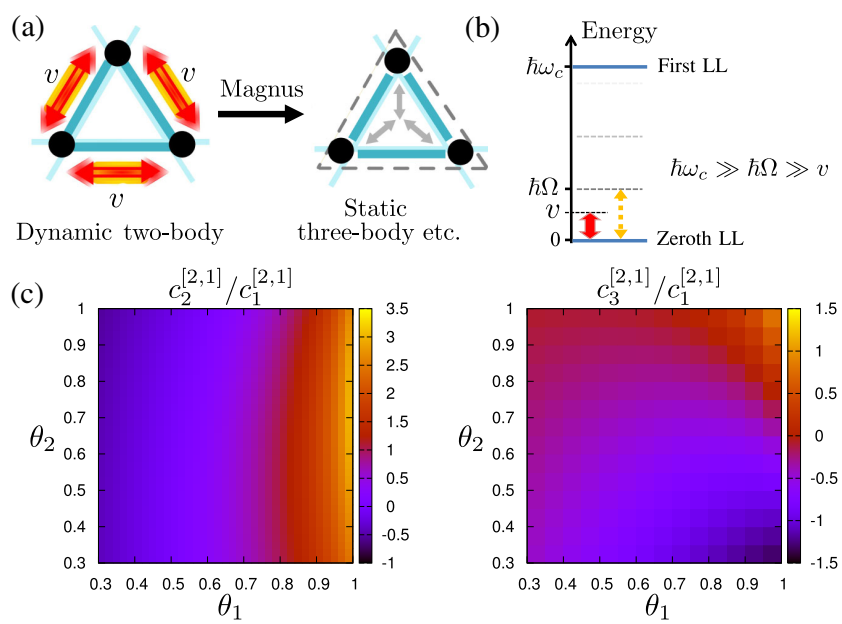

FIG. 1. (a) Time-modulated two-body interactions give rise to an effective three-body static interaction connecting different sublattices at leading order in $\Omega^{-1}$ [Eq. (6)]. (b) Energy hierarchy for the setup, with LL (or band) gap $\hbar \omega_{c}$ much larger than the driving frequency scale $\hbar \Omega$, which should also dominate the interaction $v$. (c) Three-body $\mathrm{PP}$ coefficient ratios, $c_{2} / c_{1}$ and $c_{3} / c_{1}$, for particles with opposite spins $(\lambda=[2,1])$. The driven two-body interaction is given in Eq. (7).

review now. First, one defines a relative angular momentum eigenbasis $|m\rangle$ in the LL-projected Hilbert space of $N$ particles [53] with a given permutation symmetry type $\lambda$ [50,51]. Any isotropic interaction potential $V_{\mathbf{q}}$ can be expanded in terms of PPs, $U_{m, \mathbf{q}}^{N, \lambda}$, which form a complete orthonormal basis for $N$-body operators. Below we will use the coefficients in this expansion, $c_{m}^{\lambda}$ for fixed $N=3$, in order to characterize the three-body interactions generated by the Floquet-FQH protocol. The same formalism can describe anisotropy by a redefinition of $U_{m, \mathbf{q}}^{N, \lambda} \rightarrow U_{m, \Delta m, \pm, \mathbf{q}}^{N, \lambda}$, where $\Delta m=0,2,4, \ldots$ and \pm denote the discrete symmetry $\left[U_{m, \Delta m}^{N, \lambda} \propto\left(q_{x}+i q_{y}\right)^{\Delta m}\right]$ and directionality of the anisotropic PP [32,44]. The coefficients of generalized PPs, $c_{m, \Delta m, \pm}^{N, \lambda}$, completely characterize any translation-invariant interaction and determine which FQH states are energetically favored [32]. Note that for $\Delta m=0$, anisotropic PPs reduce to the standard Haldane PPs $[6,54]$.

Floquet-FQH system.-Building on the two key inspirations above, we consider periodically driving an anisotropic FQH-FCI system such that its two-body interaction term is time modulated while the single-body term remains static, for instance by ultrafast rotation or by appropriate optical driving as detailed later,

$$
H_{\mathrm{FQH}}(t)=H_{\text {nonint }}+H_{\mathrm{int}}(t) .
$$

Here, $H_{\text {nonint }}=[1 /(2 m)] \sum_{i, \sigma} g^{a b} \Pi_{a, i}^{\sigma} \Pi_{b, i}^{\sigma}$, and the metric tensor $g^{a b}$ encodes the anisotropy $[47,55]$. We characterize 
the two-body interaction $H_{\text {int }}(t)$ with its Fourier harmonics $V_{l}$ and their momentum-space profiles $V_{\mathbf{q}}^{\sigma \sigma^{\prime}, l}$ :

$$
H_{\text {int }}(t)=\sum_{l} e^{i \Omega l t / \hbar} V_{l}=\sum_{\mathbf{q}, l, \sigma \sigma^{\prime}} e^{i \Omega l t / \hbar} V_{\mathbf{q}}^{\sigma \sigma^{\prime}, l} \bar{\rho}_{\mathbf{q}}^{\sigma} \bar{\rho}_{-\mathbf{q}}^{\sigma^{\prime}} .
$$

Let us comment on the three relevant energy scales, shown in Fig. 1(b), that are behind Eq. (3): (i) the cyclotron frequency $\hbar \omega_{c}$, set by the single-body term, (ii) the driving frequency $\hbar \Omega$, and (iii) the typical interaction strength $v$, given by the averaged $\left|V_{\mathbf{q}}^{l}\right|$. The cyclotron frequency splits the Hilbert space into energetically separated LLs, while the dynamically modulated interaction connects LLs with amplitude $v$, while simultaneously allowing energy to be absorbed or emitted in multiples of $\hbar \Omega$. To achieve interesting physics, we consider smooth (strictly low harmonic) driving obeying the hierarchy $\hbar \omega_{c} \gg$ $\hbar \Omega \gg v$, i.e., with driving being "high frequency" compared to $v$ but not to $\hbar \omega_{c}$.

The above considerations allow us to derive an effective static description of the system at stroboscopic times, such that there is approximate energy conservation and an effective long-lived ground state [56]. To see this, note that LL mixing is suppressed due to large LL gaps, high frequency driving, and the absence of high order harmonics. Hence, we obtain, via a generalized Schrieffer-Wolff transformation, an effective dynamical description of the system within each LL [44]:

$$
H_{\mathrm{FQH}}^{\mathrm{LL}}(t)=H_{\text {nonint }}+\mathcal{P} H_{\mathrm{int}}(t) \mathcal{P} \rightarrow \mathcal{P} H_{\mathrm{int}}(t) \mathcal{P}
$$

and $H_{\text {nonint }}$ drops out as an irrelevant constant.

We can further employ Eq. (2) on the effective dynamical Hamiltonian Eq. (5) to obtain the effective static description $H^{\text {eff }}$ of the system within the lowest LL. This description persists up to the exponentially long heating timescale $t_{h} \sim(\hbar / v) \exp ($ const $\times \Omega / v)$ [57-59], which is estimated to be on the order of years for the example of a cold-atom setup in Fig. 3 below. Assuming a single driving frequency $\Omega$, we have $H^{\text {eff }} \approx V_{0}+[1 /(\hbar \Omega)]\left[V_{1}, V_{-1}\right]$. Using Eq. (1), after some commutator algebra [44], we obtain $H^{\text {eff }} \approx H_{2 b}+H_{3 b}$, where the two-body term $H_{2 b}$ is the original static profile $V_{0}$ modified by an operator ordering correction [44], and the effective three-body term is

$$
\begin{aligned}
H_{3 b}= & -\frac{4}{3 \hbar \Omega} \sum_{\alpha, \beta, \gamma=\uparrow, \downarrow} \sum_{\mathbf{q}, \mathbf{q}^{\prime}} \operatorname{Im}^{-}\left\{2 V_{\mathbf{q}}^{\beta \alpha *} V_{\mathbf{q}^{\prime}}^{\beta \gamma}+V_{\mathbf{q}^{\prime}}^{\beta \gamma *} V_{\mathbf{q}-\mathbf{q}^{\prime}}^{\gamma \alpha}\right. \\
& \left.+V_{\mathbf{q}^{\prime}-\mathbf{q}}^{\alpha \gamma *} V_{\mathbf{q}}^{\beta \alpha}+V_{\mathbf{q}^{\prime}-\mathbf{q}}^{\gamma \beta *} V_{\mathbf{q}}^{\alpha \gamma}+V_{\mathbf{q}^{\prime}}^{\gamma \alpha *} V_{\mathbf{q}^{\prime}-\mathbf{q}^{\prime}}^{\alpha \beta}\right\} \\
& \times \sin \frac{\hat{\mathbf{z}} \cdot\left(\mathbf{q} \times \mathbf{q}^{\prime}\right)}{2} \bar{\rho}_{\mathbf{q}}^{\alpha} \bar{\rho}_{\mathbf{q}^{\prime}-\mathbf{q}^{\prime}}^{\beta} \bar{\rho}_{-\mathbf{q}^{\prime}}^{\gamma},
\end{aligned}
$$

where $\operatorname{Im}^{-}\left\{f_{\mathbf{q}, \mathbf{q}^{\prime}}\right\} \equiv\left(f_{\mathbf{q}, \mathbf{q}^{\prime}}-f_{-\mathbf{q},-\mathbf{q}^{\prime}}^{*}\right) /(2 i)$ and $\ell_{B}=1$.
The three-body interaction in Eq. (6) is our central result. This interaction emerges from the products of Fourier components $V_{\mathbf{q}}^{\sigma \sigma^{\prime}}$ of the original interaction, see Fig. 1(a). Because of $\mathrm{Im}^{-}$, Eq. (6) does not vanish only if $V_{\mathbf{q}}^{\sigma \sigma^{\prime}}$ (and index permutations) are complex, i.e., only if the system breaks inversion symmetry, and phase differences exist between the modulations of different interaction components. Consequently, $H_{3 b}$ is nonzero only in multicomponent anisotropic FQH systems, i.e., FCIs with multiatomic unit cells. This peculiar component dependence makes our Floquet approach particularly suited for engineering multicomponent $\mathrm{FQH}$ parent Hamiltonians. Finally, we observe that $H^{\text {eff }}$ is not constrained to be repulsive, and could be used to cancel other repulsive interaction terms in the original interaction.

Illustrative examples. - We now illustrate the versatility of the Floquet-FQH approach by some examples of interactions and many-body states it could stabilize. First, consider driving a two-body interaction $e^{i \Omega t} \sum_{\mathbf{q}} V_{\mathbf{q}} \bar{\rho}_{\mathbf{q}}^{\uparrow} \bar{\rho}_{-\mathbf{q}}^{\downarrow}+$ H.c., which consists of the simplest anisotropic PPs with $\Delta m=2[32,44]$ :

$V_{\mathbf{q}}=\cos \theta_{1} U_{0,2}+\sin \theta_{1} \cos \theta_{2} U_{1,2}+\sin \theta_{1} \sin \theta_{2} U_{2,2}$,

where $\theta_{1}, \theta_{2}$ are free parameters that keep the overall interaction strength fixed, while the prefactors of $U_{m, \Delta m}$ can be negative. Equation (7) produces a range of Floquet three-body interactions between particles with opposite spins via Eq. (6). The resulting PP coefficient ratios, $c_{2}^{[2,1]} / c_{1}^{[2,1]}$ and $c_{3}^{[2,1]} / c_{1}^{[2,1]}$, are shown in Fig. $1(\mathrm{c})$. We see that PP ratios span a wide range, and can become attractive in certain parameter regimes or strongly suppressed; e.g., $U_{1}^{3,[2,1]}$ and $U_{2}^{3,[2,1]}$ might be of comparable strength to each other and twice larger than $U_{3}^{3,[2,1]}$.

Having demonstrated the tunability of three-body Floquet PPs, we next consider two examples of exotic FQH states that they could naturally stabilize: the interlayer Pfaffian (IPf) state [60,61] and the $\nu=1$ permanent state ("111-perm") introduced in Ref. [3] (see also Ref. [62]). The IPf state is a gapped state at filling factor $\nu=2 / 3$ with non-Abelian Ising anyons, as well as spin-charge separation [63-66]. By contrast, the 111-perm state is an intriguing gapless state $[14,67-70]$ that is governed by a nonunitary conformal field theory [3,71], and represents a critical point between the integer quantum Hall ferromagnet and a paramagnet [14].

The stability of these FQH states is determined not only by the generated three-body PPs, which scale as $v^{2} / \Omega$, but also by original two-body PPs, which scale as $v$, and operator ordering corrections to them from the drive (also of the order $v^{2} / \Omega$ ) [44]. Thus, if we target a specific state, 
the original two-body interaction should be sufficiently "close" to its model interaction. Many non-Abelian FQH states can be realized in this way; e.g., the ground state of two-body PPs, $U_{1}^{[1,1]}$ and $U_{3}^{[1,1]}$, is believed to be in the Moore-Read phase [72]. In the presence of weak anisotropy, the drive could then further enhance such states by amplifying the three-body correlations, and thus the robustness of the FQH state. We now illustrate this using exact diagonalizations of continuum $\mathrm{FQH}$ systems on the sphere $[44,73]$.

For the IPf we choose the initial "hollow core" interaction consisting of two-body PPs, $U_{1}^{[2]}$ and $U_{1}^{[1,1]}$, whose strength is fixed to 1 . The dominant Floquet corrections are two-body $U_{0}^{[2]}$, and three-body $U_{1}^{N=3,[2,1]}$ and $U_{2}^{N=3,[2,1]}$. In Fig. 2(a) we show the extrapolated neutral gap of the system in the presence of these perturbations. We assume, for simplicity, that three-body PPs are of equal magnitude. The full line in Fig. 2(a) marks the value of the gap $\Delta E=0.2$, while the dashed line denotes points where the overlap of the ground state and the IPf state is equal to $90 \%$ [44]. Thus, we see that a combination of two-body and three-body Floquet terms results in the large region of a robust IPf phase with non-Abelian correlations and a large gap [top right corner of Fig. 2(a)].

Similarly, our Floquet approach is also suited for stabilizing the 111-perm state, which crucially relies on a strong $U_{1}^{[2,1]}[62,75]$. In Fig. 2(b), we fix the initial interaction to be $U_{0}^{\uparrow \downarrow}$ of magnitude 1 . The driving is assumed to generate two-body PPs $c_{1}^{[2]}=c_{1}^{[1,1]}$ and threebody PPs $c_{1}^{N=3,[2,1]}$, predominantly. By evaluating the overlap with the 111-perm state, we see that the 111-perm phase is enhanced by these perturbations, with the overlap approaching 1. At the same time, the neutral gap of the
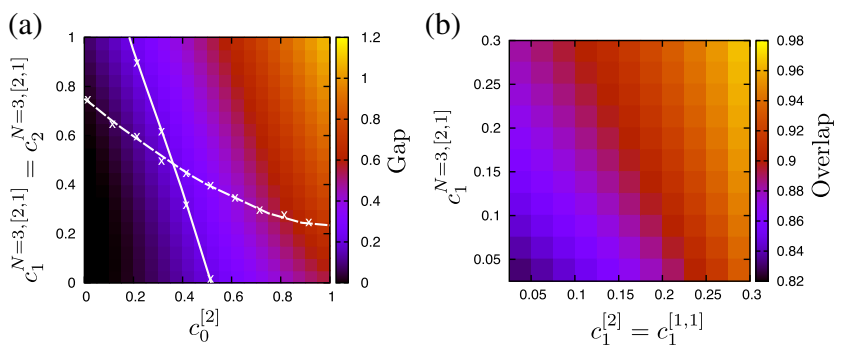

FIG. 2. (a) Extrapolated neutral gap (for system sizes $N \leq 10$ ) as a function of Floquet two-body PP, $c_{0}^{[2]}$, and three-body PPs, $c_{1}^{N=3,[2,1]}=c_{2}^{N=3,[2,1]}$. Full line traces gap $\Delta E=0.2$, while dashed line denotes values of the PPs for which the overlap of the ground state and the IPf state (for $N=10$ electrons) is $90 \%$. The robust IPf phase is stabilized in the top right corner of the phase diagram. (b) Overlap with 111-perm state is increased by a combination of Floquet two-body, $c_{1}^{[1,1]}=c_{1}^{[2]}$, and three-body $c_{1}^{N=3,[2,1]}$ perturbations. The data are for 14 electrons on the sphere. system remains very small $(\ll 1)$ throughout the phase diagram [44], which is consistent with the gapless phase in the thermodynamic limit [62]. At appropriate filling in bosonic systems, $H^{\text {eff }}$ with its tail of higher PPs may also stabilize the related 221-permanent state [75,76].

Experimental proposal.-In the continuum $\mathrm{FQH}$ case, the Floquet protocol can be implemented by modulating the component of the parallel magnetic field. For magnetic fields $B \sim 20 \mathrm{~T}$, this, however, requires a very large frequency of $\Omega \sim 1 \mathrm{THz}$. Instead, a more flexible experimental platform to implement the protocol are FCIs [77-83], which naturally possess large anisotropy, nontrivial unit cell structure, and tunable interactions [33,84-87]. We now propose a FCI model of optically driven dipolar spins, realized by trapped dipolar molecules in a 2D optical lattice, which features directional interactions that lead to a direct analogue of $[2,1]$ three-body PPs studied above in the continuum FQH case.

Each molecule in the setup possesses a rovibrational ground state, $|\downarrow\rangle=|0,0\rangle$, and three next-lowest $J=1$ states $(|1,0\rangle$ and $|1, \pm 1\rangle$ ), which are optically dressed to form a single "dark" state $|\uparrow\rangle=s|1,-1\rangle+v|1,1\rangle+w|1,0\rangle$, where $s, v$, and $w$ are rational functions of the Rabi frequencies associated with optical driving [33,44]. The $|\uparrow\rangle,|\downarrow\rangle$ states form the effective spin degrees of freedom, which are conserved when the molecules are sufficiently separated such that the physical dipole-dipole interaction between them is much weaker than the bare rotational energy (approximately the Zeeman splitting). In this case, the dipole interaction, together with a strong applied dc field that determines the quantization axis and orbital mixing, is effectively described by hardcore bosons on a lattice with the Hamiltonian [33]: $H_{\mathrm{FCI}}=-\sum_{i j} t_{i j} a_{i}^{\dagger} a_{j}+$ $\frac{1}{2} \sum_{i \neq j} V_{i j} \rho_{i} \rho_{j}$, where $a_{i}^{\dagger}=|\uparrow\rangle_{i}\left\langle\left.\downarrow\right|_{i}\right.$ is the spin-flip operator and $\rho_{i}=a_{i}^{\dagger} a_{i}$. Both the effective hopping $t_{i j}$ and Hubbard strength $V_{i j}$ originate from the same physical dipole interaction, and can be independently tuned through the $\mathbf{E}$ field and the Rabi parameters $s, v, w$ to give rise to FCI states [33].

By modulating the Rabi parameters, it is possible to keep $t_{i j}$ static while $V_{i j}$ is made time dependent. For an FCI with 2 components $A, B$, we can achieve this by dynamically modulating the Rabi parameters:

$$
\begin{aligned}
s_{A}(t) & =s_{A} e^{i \Omega_{1} t}, \quad s_{B}(t)=s_{B} e^{i \Omega_{2} t}, \\
v_{A}(t) & =v_{A} e^{i \Omega_{2} t}, \quad v_{B}(t)=v_{B} e^{i \Omega_{1} t}, \\
w_{A}(t) & =W+W^{\prime} v_{A} v_{B}^{*} e^{-i \Omega t}, \quad w_{B}(t)=W+W^{\prime} s_{A}^{*} s_{B} e^{-i \Omega t},
\end{aligned}
$$

where $\Omega=\Omega_{2}-\Omega_{1}$ sets the driving frequency, and $W=\sqrt{\Lambda(1 \mp \gamma) / 2}, \quad W^{\prime}=\sqrt{\Lambda(1 \pm \gamma) /\left(2 v_{A}^{*} v_{B} s_{A}^{*} s_{B}\right)}$, $\gamma=\sqrt{1-\left(v_{A}^{*} v_{B} s_{A}^{*} s_{B} / \Lambda^{2}\right)\left(d_{01} / d_{00}\right)^{4}}$, with $\Lambda$ a real tuning parameter and $d_{01}=\left\langle 1, \pm 1\left|d_{z}\right| 0,0\right\rangle, d_{00}=\left\langle 1,0\left|d_{z}\right| 0,0\right\rangle$ dipole transition matrix elements that depend on the applied 


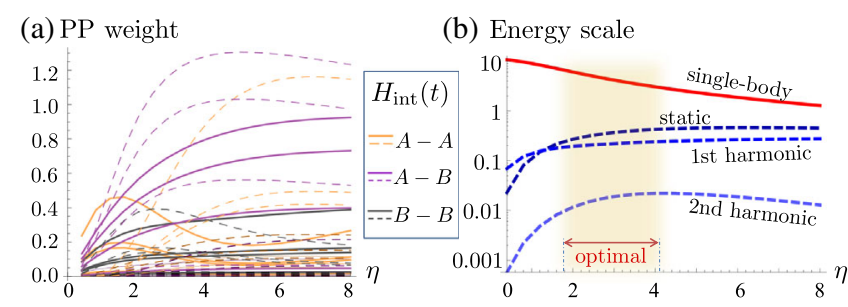

FIG. 3. (a) Coefficients of two-body PPs $c_{1}, c_{2}, c_{3}$, and $c_{4}$ as a function of $\eta$ for illustrative parameters yielding a band of flatness $\approx 3$ [44]. Solid (dashed) curves represent dynamic (static) contributions, which are colored according to whether they act between $A A, A B$, or $B B$ sites. (b) Comparison between the energy scales of the single-body, static twobody $(v)$ and lowest two harmonics of the dynamic two-body parts of $H_{\mathrm{FCI}}(t)$ for different $\eta$. For all purposes, the second harmonic can be neglected. In the optimal shaded regime we have $v \ll \hbar \Omega \ll \hbar \omega_{c}$.

E field. The Rabi parameter magnitudes are chosen to optimize the band flatness of the resultant tight-binding FCI Hamiltonian [44], leaving a dynamic two-body interaction with a single tunable parameter $\eta=2 E I d / \hbar^{2}$, the ratio of the molecular dipole energy $E d$ to its rotational energy scale $\hbar^{2} / 2 I, I$ being the moment of inertia. Coefficients of various two-body PPs are plotted as a function of $\eta$ in Fig. 3(a), and we see that interactions between $A$ and $B$ sites (purple) dominate for most $\eta$. For very small $\eta$, the interaction is mostly dynamical, and its rapid sign fluctuations may destabilize the Floquet ground state. The relevant energy scales are shown in Fig. 3(b). In the optimal regime, $1.5 \lesssim \eta \lesssim 4$, the single-body hoppings (and hence gap) are one to two orders larger than the interaction, thereby satisfying the requisite hierarchy $v \ll \hbar \Omega \ll \hbar \omega_{c}$. At the same time, the static interaction between sublattices is still larger than the dynamic part. Thus, for $\eta \approx 3$ we achieve a direct analog of the above $U_{m}^{[2,1]}$ three-body interaction, assuming we are in the thermodynamic limit where the GMP algebra is valid. Away from this limit, details of the Bloch wave functions, interband transitions, and imperfections of the band flatness [88] could affect the stability of the Floquet FCI state.

Conclusions.-We have presented an approach for generating multicomponent three-body $\mathrm{FQH}-\mathrm{FCI}$ interactions, Eq. (6), via driving anisotropic two-body interactions with inhomogeneous phase offsets. Our approach is valid in the regime $v \ll \hbar \Omega \ll \hbar \omega_{c}$, and yields an effective three-body interaction whose magnitude scales like $\Omega^{-1}$, rather than the conventional $\omega_{c}^{-1}$ due to LL mixing. The approach relies on the unique properties of the GMP algebra, and thus applies to both FQH and FCI systems in the thermodynamic limit. We have demonstrated that this approach provides a new route for the exploration of both gapped and gapless multicomponent non-Abelian $\mathrm{FQH}$ states, and proposed its implementation in a Floquet FCI of optically dressed dipolar molecules, where time reversal is broken by the asymmetry between the left- and right-circularly polarized optical driving.

Statement of compliance with EPSRC policy framework on research data: This publication is theoretical work that does not require supporting research data.

We thank Nie Wei, Nicolas Regnault, and F.D.M. Haldane for helpful discussions. W. W. H. is supported by the Gordon and Betty Moore Foundations EPiQS Initiative through Grant No. GBMF4306. J. G. is supported by Singapore Ministry of Education Academic Research Fund Tier I (WBS No. R-144-000-353-112) and by the Singapore NRF Grant No. NRF-NRFI2017-04 (WBS No. R-144-000-378-281). Z. P. acknowledges support by EPSRC Grants No. EP/P009409/1 and No. EP/R020612/1.

[1] M. Freedman, A. Kitaev, M. Larsen, and Z. Wang, Bull. Am. Math. Soc. 40, 31 (2003).

[2] C. Nayak, S. H. Simon, A. Stern, M. Freedman, and S. D. Sarma, Rev. Mod. Phys. 80, 1083 (2008).

[3] G. Moore and N. Read, Nucl. Phys. B360, 362 (1991).

[4] A. Kitaev, Ann. Phys. (Amsterdam) 321, 2 (2006).

[5] N. Read and D. Green, Phys. Rev. B 61, 10267 (2000).

[6] R. Prange and S. Girvin, The Quantum Hall Effect, Graduate Texts in Contemporary Physics (Springer-Verlag, Berlin, 1987).

[7] R. Willett, J. P. Eisenstein, H. L. Störmer, D. C. Tsui, A. C. Gossard, and J.H. English, Phys. Rev. Lett. 59, 1776 (1987).

[8] D.-K. Ki, V. I. Fal'ko, D. A. Abanin, and A. F. Morpurgo, Nano Lett. 14, 2135 (2014).

[9] A. Zibrov, C. Kometter, H. Zhou, E. Spanton, T. Taniguchi, K. Watanabe, M. Zaletel, and A. Young, Nature (London) 549, 360 (2017).

[10] R. H. Morf, Phys. Rev. Lett. 80, 1505 (1998).

[11] E. H. Rezayi and F. D. M. Haldane, Phys. Rev. Lett. 84, 4685 (2000).

[12] M. Storni, R. H. Morf, and S. Das Sarma, Phys. Rev. Lett. 104, 076803 (2010).

[13] M. Greiter, X. Wen, and F. Wilczek, Nucl. Phys. B374, 567 (1992).

[14] N. Read and E. Rezayi, Phys. Rev. B 54, 16864 (1996).

[15] M. Barkeshli and X.-G. Wen, Phys. Rev. B 82, 245301 (2010).

[16] E. Ardonne, Phys. Rev. Lett. 102, 180401 (2009).

[17] M. Barkeshli and X.-G. Wen, Phys. Rev. B 82, 245301 (2010).

[18] W. Bishara and C. Nayak, Phys. Rev. B 80, 121302 (2009).

[19] M. R. Peterson and C. Nayak, Phys. Rev. B 87, 245129 (2013).

[20] I. Sodemann and A.H. MacDonald, Phys. Rev. B 87, 245425 (2013).

[21] S. H. Simon and E. H. Rezayi, Phys. Rev. B 87, 155426 (2013).

[22] A. Ghazaryan, T. Graß, M. J. Gullans, P. Ghaemi, and M. Hafezi, Phys. Rev. Lett. 119, 247403 (2017). 
[23] J. Cayssol, B. Dóra, F. Simon, and R. Moessner, Phys. Status Solidi RRL 7, 101 (2013).

[24] M. Bukov, L. D’Alessio, and A. Polkovnikov, Adv. Phys. 64, 139 (2015).

[25] A. Eckardt, Rev. Mod. Phys. 89, 011004 (2017).

[26] C. H. Lee, G. Li, G. Jin, Y. Liu, and X. Zhang, Phys. Rev. B 97, 085110 (2018).

[27] B. Wang, F. N. Ünal, and A. Eckardt, Phys. Rev. Lett. 120, 243602 (2018).

[28] L. Li, C. H. Lee, and J. Gong, Phys. Rev. Lett. 121, 036401 (2018).

[29] S. M. Girvin, A. H. MacDonald, and P. M. Platzman, Phys. Rev. Lett. 54, 581 (1985).

[30] S. M. Girvin, A. H. MacDonald, and P. M. Platzman, Phys. Rev. B 33, 2481 (1986).

[31] S. A. Parameswaran, R. Roy, and S. L. Sondhi, Phys. Rev. B 85, 241308 (2012).

[32] B. Yang, Z.-X. Hu, C. H. Lee, and Z. Papić, Phys. Rev. Lett. 118, 146403 (2017).

[33] N. Y. Yao, A. V. Gorshkov, C. R. Laumann, A. M. Läuchli, J. Ye, and M. D. Lukin, Phys. Rev. Lett. 110, 185302 (2013).

[34] A. G. Grushin, Á. Gómez-León, and T. Neupert, Phys. Rev. Lett. 112, 156801 (2014).

[35] E. Anisimovas, G. Žlabys, B. M. Anderson, G. Juzeliūnas, and A. Eckardt, Phys. Rev. B 91, 245135 (2015).

[36] J. Klinovaja, P. Stano, and D. Loss, Phys. Rev. Lett. 116, 176401 (2016).

[37] H. H. Yap, L. Zhou, C. H. Lee, and J. Gong, Phys. Rev. B 97, 165142 (2018).

[38] G. Jotzu, M. Messer, R. Desbuquois, M. Lebrat, T. Uehlinger, D. Greif, and T. Esslinger, Nature (London) 515, 237 (2014).

[39] J. Gong, L. Morales-Molina, and P. Hänggi, Phys. Rev. Lett. 103, 133002 (2009).

[40] Á. Rapp, X. Deng, and L. Santos, Phys. Rev. Lett. 109, 203005 (2012).

[41] S. Greschner, G. Sun, D. Poletti, and L. Santos, Phys. Rev. Lett. 113, 215303 (2014).

[42] F. Meinert, M. J. Mark, K. Lauber, A. J. Daley, and H.-C. Nägerl, Phys. Rev. Lett. 116, 205301 (2016).

[43] B. A. Bernevig and N. Regnault, Phys. Rev. B 85, 075128 (2012).

[44] See Supplemental Material at http://link.aps.org/ supplemental/10.1103/PhysRevLett.121.237401 for details on the statements and calculations presented in the main text.

[45] D. Burum, Phys. Rev. B 24, 3684 (1981).

[46] S. Blanes, F. Casas, J. Oteo, and J. Ros, Phys. Rep. 470, 151 (2009).

[47] B. Yang, Z. Papić, E. H. Rezayi, R. N. Bhatt, and F. D. M. Haldane, Phys. Rev. B 85, 165318 (2012).

[48] Z. Papić, Phys. Rev. B 87, 245315 (2013).

[49] B. Yang, C. H. Lee, C. Zhang, and Z.-X. Hu, Phys. Rev. B 96, 195140 (2017).

[50] S. H. Simon, E. H. Rezayi, and N. R. Cooper, Phys. Rev. B 75, 195306 (2007).

[51] S. C. Davenport and S. H. Simon, Phys. Rev. B 85, 075430 (2012)

[52] C. H. Lee, R. Thomale, and X.-L. Qi, Phys. Rev. B 88, 035101 (2013).
[53] A. H. Macdonald, arXiv:cond-mat/9410047.

[54] F. D. M. Haldane, Phys. Rev. Lett. 51, 605 (1983).

[55] F. D. M. Haldane, Phys. Rev. Lett. 107, 116801 (2011).

[56] W. W. Ho and D. A. Abanin, arXiv:1611.05024.

[57] D. Abanin, W. De Roeck, W. W. Ho, and F. Huveneers, Commun. Math. Phys. 354, 809 (2017).

[58] D. A. Abanin, W. De Roeck, W. W. Ho, and F. Huveneers, Phys. Rev. B 95, 014112 (2017).

[59] T. Mori, T. Kuwahara, and K. Saito, Phys. Rev. Lett. 116, 120401 (2016).

[60] E. Ardonne, F. J. M. van Lankvelt, A. W. W. Ludwig, and K. Schoutens, Phys. Rev. B 65, 041305 (2002).

[61] M. Barkeshli and X.-G. Wen, Phys. Rev. B 82, 233301 (2010).

[62] D. Green, Ph.D. thesis, Yale University, 2001, https://arxiv .org/abs/cond-mat/0202455.

[63] E. Ardonne, E. J. Bergholtz, J. Kailasvuori, and E. Wikberg, J. Stat. Mech. (2008) P04016.

[64] S. Geraedts, M. P. Zaletel, Z. Papić, and R. S. K. Mong, Phys. Rev. B 91, 205139 (2015).

[65] M. R. Peterson, Y.-L. Wu, M. Cheng, M. Barkeshli, Z. Wang, and S. Das Sarma, Phys. Rev. B 92, 035103 (2015).

[66] Z. Liu, A. Vaezi, K. Lee, and E.-A. Kim, Phys. Rev. B 92, 081102 (2015).

[67] F. D. M. Haldane and E. H. Rezayi, Phys. Rev. Lett. 60, 956 (1988).

[68] S. H. Simon, E. H. Rezayi, N. R. Cooper, and I. Berdnikov, Phys. Rev. B 75, 075317 (2007).

[69] B. Andrei Bernevig and F. D. M. Haldane, Phys. Rev. Lett. 100, 246802 (2008).

[70] Z. Papić, Phys. Rev. B 90, 075304 (2014).

[71] N. Read, Phys. Rev. B 79, 245304 (2009).

[72] M. R. Peterson, K. Park, and S. Das Sarma, Phys. Rev. Lett. 101, 156803 (2008).

[73] The Supplemental Material [44] also discusses their entanglement spectrum signatures [74].

[74] H. Li and F. D. M. Haldane, Phys. Rev. Lett. 101, 010504 (2008).

[75] C. H. Lee, Z. Papić, and R. Thomale, Phys. Rev. X 5, 041003 (2015).

[76] E. Ardonne and N. Regnault, Phys. Rev. B 84, 205134 (2011).

[77] T. Neupert, L. Santos, C. Chamon, and C. Mudry, Phys. Rev. Lett. 106, 236804 (2011).

[78] K. Sun, Z. Gu, H. Katsura, and S. Das Sarma, Phys. Rev. Lett. 106, 236803 (2011).

[79] N. Regnault and B. A. Bernevig, Phys. Rev. X 1, 021014 (2011).

[80] E. J. Bergholtz and Z. Liu, Int. J. Mod. Phys. B 27, 1330017 (2013).

[81] C. H. Lee and X.-L. Qi, Phys. Rev. B 90, 085103 (2014).

[82] M. Claassen, C. H. Lee, R. Thomale, X.-L. Qi, and T. P. Devereaux, Phys. Rev. Lett. 114, 236802 (2015).

[83] C. H. Lee, M. Claassen, and R. Thomale, Phys. Rev. B 96, 165150 (2017).

[84] N. Y. Yao, C. R. Laumann, A. V. Gorshkov, S. D. Bennett, E. Demler, P. Zoller, and M. D. Lukin, Phys. Rev. Lett. 109, 266804 (2012). 
[85] D. Peter, N. Y. Yao, N. Lang, S. D. Huber, M. D. Lukin, and H. P. Büchler, Phys. Rev. A 91, 053617 (2015).

[86] M. F. Maghrebi, N. Y. Yao, M. Hafezi, T. Pohl, O. Firstenberg, and A. V. Gorshkov, Phys. Rev. A 91, 033838 (2015).
[87] N. Y. Yao, S. D. Bennett, C. R. Laumann, B. L. Lev, and A. V. Gorshkov, Phys. Rev. A 92, 033609 (2015).

[88] S. Kourtis, T. Neupert, C. Chamon, and C. Mudry, Phys. Rev. Lett. 112, 126806 (2014). 